\title{
Téoros
}

Revue de recherche en tourisme

\section{Interventions touristiques au Québec : chronologie des décisions marquantes situées dans le contexte historique}

\section{Louis Jolin et Jean-Marc Descôteaux}

Volume 14, numéro 2, été 1995

Le tourisme : toute une histoire!

URI : https://id.erudit.org/iderudit/1075101ar

DOI : https://doi.org/10.7202/1075101ar

Aller au sommaire du numéro

Éditeur(s)

Université du Québec à Montréal

ISSN

0712-8657 (imprimé)

1923-2705 (numérique)

Découvrir la revue

Citer cet article

Jolin, L. \& Descôteaux, J.-M. (1995). Interventions touristiques au Québec : chronologie des décisions marquantes situées dans le contexte historique.

Téoros, 14(2), 36-41. https://doi.org/10.7202/1075101ar d'utilisation que vous pouvez consulter en ligne. 


\section{Interventions touristiques au Québec : chronologie des décisions marquantes situées dans le contexte historique ${ }^{(1)}$}

Louis Jolin et Jean-Marc Descôteaux*

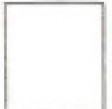

Cet article, composé essentiellement d'un tableau et d'une brève analyse des tendances, vise à mettre en lumière de façon chronologiquelesprincipales interventions et décisions publiques en matière de tourisme au Québec. Le tableau ${ }^{(2)}$ découpe l'histoire du Québec de 1919 à nos jours en 6 grandes périodes touristiques dans lesquelless s'insèrentles diverses interventions de l'État.

En référence aux principales actions de l'État, quelques tendances sont soulignées, parfois de façon interrogative, en rapport avec les axes suivants: le tourisme comme phénomène économique, la démocratisation de la pratique touristique, la régionalisation du tourisme, la protection du consommateur, la professionnalisation du tourisme, la consultation et la concertation des partenaires, le tourisme comme secteur d'intervention de l'État.

\section{DÉCISIONS ET ACTIONS DES GOUVERNEMENTS AU COURS DES GRANDES PÉRIODES DU TOURISME AU QUÉBEC (DE 1919 A AUJOURD'HUI) ${ }^{\text {(3) }}$}

I.

1926

1927

1932

1933

1934

1940

II =

1946

1960

1961

1963

III -

1965

1967

Monsieur Louls Jolin est directeur du module de gestion du tourisme et de thotellerie et protesseur au dépariement d'etudes urbaines et tourstiques a I"Universite du Quebec a Montreal. Monseur Jean-Marc Descolleaux est charge de cours au département d'etudes urbanes et fouristiques a l'Unversne du Ouebec a Montreal

\section{LA PÉRIODE D'AVANT-GUERRE OU LA NAISSANCE DU TOURISME AU QUÉBEC (1919-1945)}

Création d'un Office de tourisme et des congrès à Montráal, ancêtre de l'OCTGM actuel, l'un des plus vieux offices de tourisme en Amérique du Nord.

Premictre carte officielle du résesu routier de la province.

Pulblication du guide touristique Voyez Québec d'abord - See Quebec First par le nouveau service du tourisme du ministère de la Voirie.

Ouverture de bureaux de renseignements pour les touristes a Montrál et a Quéthec.

Création d'un premier Conseil du tourisme. À cette époque, il n'y a pas de politique prócise du tourisme.

Críation de l'Office de tourisme du Canada.

Ouverture de l'aéroport international de Dorval et ouverture d'une première délégation du Québec à New York

\section{LA PHASE DE MÛRISSEMENT (1945-1965)}

Création de l'Office provincial de publicité; l'ancien service du tourisme en devient l'un des secteurs les plus importants.

L'Office provincial de publicité passe sous la juridiction du Secrétariat de la Province. Dans les annćes 1960, on assistera a la création de grandés institutions publiques à caractère économique dont la Société de développement industriel (SDI) et la Caisse de dépồt et de placement.

Le service du tourisme devient une entité distincte et prend le nom d'Office du tourisme de la province de Québec. Renaissances du Conseil du tourisme, à titre d'organisme consultatif. La priorité touristique se limitait alors a la publicité et on ne se précoccupait guère du produit.

Un jalon umportant avec la création du ministère du Tourisme, de la Chasse et de la Pèche (MTCP).

Loi sur / Tötellerie (cette loi abrogeait la Loi de finspection des bótels et la section II de la Lai des liances).

\section{LA CROISSANCE TOURISTIQUE (1965.1975)}

Mises en place d'éléments attractifs (parcs, campings, haltes routières) en vue d'Expo 67 (exposition universelle de Montréal) qui marque l'ouverture du Quethec sur le monde. Il y a une grande évolution du tourisme et du produit touristique, c'est la période glorieuse du tourisne au Québec.

Ébuche d'une politique, Le tourisme au Québec: programme d'études préalables a l'élaboration d'une politique du tourisme au Québec, préparce par SOTAR pour le compte du MTCP.

1968 Adoption de la Loi nar /Ofice de plasifiarion du Québec (OPQ) dont le mandat inclut des responsabilités en matière de développement régional; cette loi est moslifiée en 1969 par la création de l'Office de planification et de développement du Quéthec (OPDQ); elleconfere au nouvelorganisme des responsabilités cn matiěre de développernent. 
Fondation de l'Institut de tourisme et d'hôtellerie du Québec (TTHQ) et création de l'Office franco-québécois pour la jeunesse (OFQJ).

Annonce par le gouvernement fédéral de la mise en chantier d'un deuxiène aéroport international dans la région de Montréal.

Loi sar la conscruation de la faune, notamment modifiće en 1978 pour donner naissance aux zones d'exploitation contrôlée (ZEC).

1972 Loi sur les tranports (qui abroge la Loi de la Rigie des transports) modifiée à plusieurs reprises et complétée par divers règlements.

Loisur lcs birn culturels (modifiée plusieurs fois, notamment en 1978 et en 1985). Elle vise à protéger les biens culturels qui présentent un intérêt public, historique et qui sont un témoignage de notre passé.

1974 Une première stratégie de développement touristique: le CPDQ (Conseil de planification et de développément du Québec), mandaté par le gouvernement, dépose un document en six volumes intitulé Vers une industrie touristique au Québec: ếléments d'une stratégie de développement. Malheureusement, on n'a jamais entendu parler par la suite de ce document.

Loi sur les agents de voyager, modifiée en 1977, en 1979, en 1981, en 1990 et en 1992 notamment pour accentuer son caractère de protection du consommateur; depuis 1981, la loi est sous la responsabilité effective du président de l'Office de la protection du consommateur.

IVLA MATURITÉ (1975-1985)

Création de la Régie des insta llations olympiques (RIO) dans le but d'assurer la gestion et de parachever les travaux du Stade en vue des Jeux olympiques de 1976, Ja Ville de Montréal s'avérant incapable de mener à terme le dossier. Les Jeux olympiques donnent au secteur du tourisme un regain d'énergie. Inauguration de l'aéroport international de Mirabel.

\section{8} d'un réseau de parcs sur une base juridique cohérente. Les municipalités et les nouvelles municipalités régionales de comté (MRC) jouent un rôle décisif; la Loi sur Taménagement et luarbamisme confie la responsalvilité de l'aménagement du territoire aux MRC.

La Conférence sectorielle sur le tourisme (aussi appelée premier Sommet touristique) tenue à Sherbrooke marque les débuts du crédit touristique (formule d'aide à l'entreprise) et l'officialisation des associations touristiques régionales (ATR): le Québec est ainsi découpé en 18 régions touristiques. Le tourisme, perspectives de relance est un document de réflexion préparé par le Secrétariat des conférences socio-économiques dans le cadre de la Conférence sectorielle sur le tourisme. Les travaux de la Conférence, à partir dudit document de réflexion, ont eux-mêmes pavé la voie a la stratégie préconiséc en 1979 dans l'énoncé de politique économique du gouvernement, Bàtir le Québec (chapitre 16 sur le tourisme).

Création à l'Université du Quéthec à Montréal (UQAM) de la première formation universitaire en tourisme au Quéhec (baccalauréat en gestion et intervention touristiques).

Création au niveau fédéral de la Société de la Couronne, Via Rail: fusion des services ferroviaires passagers du Canadien National (CN) et du Canadien Pacifique (CP).

1979 Une politiqueculturelle attendue, préparée sous l'égide du ministre des Affaires culturelles, entre en vigueur de même qu'une politique du loisir publiée dans On a un monde à recrécr, livre blanc sur le loisir au Quetbec.

Loisur laile au diveloppenuent touristigue.

Planification d'un développement fondé sur des circuits, des corridors, des produits touristiques intégrés, des zones de developpement. Injoction massive de $S$ dee la part des deux paliers de gouvernement dans l'amélioration du domaine skiahle. Globalement, on note danscette phase de wmaturités une amélioration de l'offre qui a le mérite d'empêcher la décroissance touristique.

Le MTCP est aboli. On décide de rattacher le tourisme, considéré comme une industrie, au ministère de l'Industrie, du Commerce et du Tourisme (MICT). 
Le Haut-Commissariat à la jeunesse, aux loisirs et aux sports cède également sa place au nouveau ministère du Loisir, de la Chasse et de la Péche (MLCP), qui sera chargé des dossiers dits de aloisir touristique* ou wtourisme social*. Le Service canadien des Parcs (Pares Canada) est rattaché a Environnement Canada. Tourisme Canada (pour l'Office de tourisme du Canada, crée en 1934) relève du ministère de l'Expansion économique régionale (MEER). Entrée en vigueur du programmed action économique Bàtir le Québec, phase II (Le virage technologique) qui poursuit fondamentalement la stratégie mise de l'avant dans Bàtir le Québec.

1983 Lot sur la conervation et la mise en waleur de la faume qui abroge la Loi sur la conterwation de la fanone et vise à protéger, conserver, mettre en valeur et gérer la faune (certaines dispositions de la loi ont trait a la mise sur pied de la Fondation quétbécoise de la faune). La loi régit les territoires structurés terres domaniales, pourvoiries, zones d'exploitation contrôlée et refuges fauniques.

Ouverture du Palais des congrèts de Montréal.

Création d'un ministère du Tourisme autonome et autre ébauche d'une politique du tourisme, Le tourisme au Québec, bilan et perspectives d'action 1985-1988, qui pousse plus loin la réflexion amorcée dans Bàtir le Qućbec.

Création de la Sociêté des établissements de plein air du Québec (SEPAQ), relevant du MLCP. Son mandat: rentabiliser la gestion d'équipements et de territoires - tel le Mont Ste-Anne - appartenant au gouvernement du Québec.

Dossierschauds à cette époque: les sagas de Québecairet du Manoir Richelieu qui seront la propriété du gouvernement du Québec pendant quelque temps, sous le régime péquiste, avant qu'ils ne redeviennent des entreprises privées dans les premiers mois du gouvernement Bourassa.

Nomination au fédéral d'un ministre d'Êtat au Tourisme, relevant du «nouweaus ministère de l'Expansion industrielle régionale (MEIR). Le ministre d'État est un ministre junior sans portefeuille; son rôle consiste à conseiller le ministre du MEIR sur les questions relatives aux politiques et a l'exploitation touchant l'expansion et la promotion du tourisme au Canada.

On commence à parler de l'éventuelle privatisation de sociétés d'État (souvent deficitaires) telles que Air Canada, Via Rail et certaines administrations aéroportuaires conadiennes.

En vue de revitaliser les aménagements des principaux ports canadiens, création au Québec des sociétés du Vieux-Port de Montréal et du Vieux-Port de Québec, sociétés ayant pour mandat la gestion, l'aménagement et la mise en valeur des territoires ainsi désignés.

\section{LA STABILITÉ (1985-1992)}

Le ministère du Tourisme a son propre budget; on reconnait donc maintenant zu tourisme ses lettres de noblesse. Fonctionnement du secteur mieux articulé par la présence et l'action soutenue des ATR. Mise sur pied par le ministère et les ATR des Grands Prix du tourisme québécois afin de souligner et de valoriser l'excellence touristique au Québec. La RIO, I'TTHQ et la Société du Palais des congrès de Montréal relevent directement du ministre du Tourisine.

Processus de discussion et de consultation mis de l'avant au niveau fédéral par le nouveau ministre d'État au Tourisme, Tom McMillan, autour du document de réflexion Le tourisme de demain. Pour une stratégie canadienne du tourisme.

Signature d'une entente auxiliaire Canada-Québec sur le tourisme (1985= $1900)$, entre les gouvernements fédéral et provincial, laquelle entente prévoit des investissements de l'ordre de $100 \mathrm{M}$ S sur cinq ans. Cette entente héneficiera beaucoup aux centres tet infrastructures de ski alpin. Alwolition du Conseil quéthécois du tourisme (CQT), organisme consultatif qui avait pour mandat de conseiller les ministres responsables. 

sur (bö́rilkrie et a un champ d'application plus large).

Entrée en vigueur d'une nouvelle politique de signalisation touristique au Québec (les panneaux bleus avec pictogrammes), mise de l'avant par le ministère du Tourisme et le ministère des Transports du Québec, en collaboration avec les ATR.

1988 La Loi sur l'aide au developpement touristique devient presque inopérante. Les programmes d'aide financière sont établis parvoie réglementaire en vertu de la Loi sur la Société de développement industriel.

Nouveau document élaboré par le ministère du Tourisme: Stratégie et politiques d'orientation en matière touristique, complété en $1989 \mathrm{par}$ Opérations du tourisme québécois.

Signature d'une entente auxiliaire Canada-Québec sur le développement économique des régions du Québec (EDER).

L'Office national des transports (ONT) prend la reléve de la Commission canadienne des transports et est chargée d'appliquér la politique nationale sur les transports conformément a l'esprit de la Loi nationale sur les tranports (1987) qui ouvre la voie à une certaine déréglementation de ce secteur. On va de l'avant avec la privatisation d'Air Canada, société de la Couronne. Nouvelle approche marketing par produits mise de l'avant par le Ministère. Une $19^{\mathrm{r}}$ région touristique est reconnue: Laval.

Tourisme Canada relevve maintenant du nouveau ministère Industrie, Sciences et Technologie-Canada (ISTC).

1990 Entrée en vigueur de la politique touristique fédérale Le tourisme, un pas à franchir, tlaborée, sous la direction du ministre d'Etat (petites entreprises et tourisme), par ISTC.

1991 Création du Buresu fédéral de développement régional (BFDR)-Québec dont le mandat est de promouvoir le développement économique des régions du Québec.

1992 La fin de cette période voit l'entrée en vigueur de la première vraie politique articulée du tourisme au Québec, Des saisons et des gens - le partenariat, force motrice de l'industrie touristique. Énoncé de politique et plan d'action en matière de tourisme. On y réaffirme l'accent mis sur un positionnement aNature-Cultures, la stratégie marketing portant sur une offre touristique bascé sur huit produits distinctifs, la consolidation des marchés déjà visés et la prospection de *marchés en développemento de même qu'un partenariat renouvelé gouvernement-associations-entres privéess.

Le ministre de l'Industrie et du Commerce (MIC) dévoile sa stratégie économique pour le Québec, celle des egrappes industrielles*. Le tourisme sera identifié comme le quatorzième secteur pouvant faire l'objet d'une agrappe industrielle* reconnue.

Le Secréta riat aux affaires régionales (SAR) est créé et remplace l'OPDQ qui cessera toutes ses activités en 1993, après 25 ans d'existence. Le SAR découle de la nouvelle politique gouvernementale en matière de développernent régional qui accentue la décentralisation desprogrammes de développement. Cela crée une nouvelle dynamique pour le développement du tourisme au niveau régional avec le rôle prépondérant que les conseils réggionaux de développement (CRD) se voient confier à la suite de la réforme Picotte. Entrée en vigueur de la politique de cession de l'exploitation de certains aéroports canadiens, de Transports Canada à des entités constituées en sociétés privées sans but lueratif. Aéroports de Montréal (ADM), créée dès 1989, devient en 1992 la société gestionnaire des aéroports de Dorval et de Mirabel. D'autres infrastructures aéroportuaires, ailleurs au pays, sont alors cédées ou en voie de l'être à des sociétés privées de gestion.

Signature d'une nouvelle entente auxiliaire Canada-Québec sur le tourisme (1992-1997), laquelle prévoit des investissements de l'ordre de 100 M S sur cinq ans. 


\section{VI- Nouveaux horizons (1993-... $)^{(4)}$}

Le recul historique fait défaut pour apprécier les décisions et les faits marquants de cette nouvelle période que nous dénommons *nouveaux horizons" pour le moment. Les changements de gouvernements au fédếral comme au Québec ne sont pas les moindres de oes faits et ils ont eu et auront des incidences dont on mesure enoore mal la portée actuellement. Voic quelques-uns des faits récents:

- Création de la Société des casinos du Québec et ouverture du Casino de Montréal (octobre 1993) et de celui de Charlevoix (juin 1994). Ouverture prochaine de celui de l'Outaouais.

- Adoption de la Loi sur la Régie de alcools, des courses et des jeux (deux régies sont fusionnées).

- Nouvelle Société du Centre des congrès de Québec qui relève du ministre responsable du Tourisme (1993). Construction du Centre des congrès de Québec.

- Lancement par le gouvernement Chrétien du programme - Travaux et infrastructures" qui constitue une source de financernent pour quelques projets de nature touristique (le nouveau Centre des congrès de Québec, le Centre de villégiature de Tremblant (Intrawest)).

- Tourisme Canada devient, en juin 1993, une sous-direction du ministère Industrieet Sciences Canada avant de devenir une sous-direction de Industrie-Canada pour être remplacé à toutes fins pratiques par la nouvelle Commission canadienne du tourisme (1995).

- LeService canadien des parcs estintégré au nouveau ministère du Patrimoine canadien.

- LeministèreduTourismeduQuébec disparaît comme entité ministérielle autonome, sous le gouvernement Johnson, pour être intégré au ministère de l'Industrie, du Commerce, de la Science et de la Technologie. Un projet de loi créant la Sociéré québécoise du tourismeest sanctionné le 17 juin 1994 mais n'est jamais entré en vigueur. Le nouveau gouvernement péquiste n'entend pas le rendre envigueur et Tourisme Québec fonctionne, dans les faits, comme une entité autonome sous la responsabilité effective de la ministre déléguée au Tourisme.
- Leministère du Loisir, dela Chassect de la Pêche n'existe plus: certains dossiers (loisir) relèvent du ministère des Affaires municipales et les autres (faune, parcs) du nouveau ministčre de l'Environnement et de la Faune.

- La stratégie des grappes industrielles ne semble plus être maintenue. Un nouveau plan d'action de Tourisme Québec devrait être rendu public sous peu. Une réflexion est en cours quant à la revitalisation de la concertation gouvernement-industrie.

\section{Quelques tendances... un questionnement pour l'avenir}

Pour les fins du présent article, il nous semble pertinent, en référence à un certain nombre d'actions (énoncés, lois, programmes, investissements, etc.) de dégager quelques grandes tendances, souvent de manière interrogative, selon un certain nombre d'axes.

\section{Le tourisme comme phénomène économique}

L'affirmation de l'importance du tourisme comme phénomène économique devient le leiunotiv des déclarations et énoncés politiques des dernières années et semble justifier, à elle seule, l'interventiondel'État. N'est-ce pas un peu réducteur? Par contre, même si les budgets de l'Etat, dans le secteur du tourisme, ont augmenté(surtout au plan dela promotion), la part budgétaire consacrée au ministère responsable du tourisme (moins de $1 \%$ ) ne semble jamais correspondre à l'importance du tourisme comme phénomène économique. Certes, il faut considérer l'intervention budgétaire globale, c'est-à-dire tous les crédies, concernant directement ou indirectement le tourisme, géré́s par d'autres ministères selon leurs propres objectifs. Néanmoins, la faiblesse relatuve des budgets étatiques ne traduit-elle pas une position plus fondamentale de l'État à l'égard du tourisme: c'est un secteur économique qui doit évoluer le plus possible selon les règles du jeu du libêralisme économique, mểme si certaines caractéristiques du phénomène touristiquenécossitentune action de régulation de l'Etat?

\section{La démocratisation du tourisme}

Cette préoccupation de l'accessibilité aux ressources, de la démocratisation de la pravique touristique n'a jamais été un élément marquant de la politique officielle du tourismeou de l'action du ministère responsable du tourismemais elle fut successivement celle, selon les années, du Haut Commissariat à la jeunesse, aux loisirs et aux sports et du ministère du Loisir, de la Chasse et de la Péche. Une dichotomie s'est alors installée: le tourisme à vocation économique, commercale, au ministère du Tourisme ou au ministère de l'Industrie et du Commerce vs le loisir touristique, le «tourisme socials au ministère du Loisir. Mais à la faveur de la crise des finances publiques, cette préoccupation d'un *tourisme social w semble perdre nettement du terrain: les subventions au loisir touristique ou au tourismesocial ont diminué radicalement, l'ère est maintenant à la tarification des services aux usagers et à la rentabilisation des équipements. Pourtant, l'accessibilité aux vacances et au tourisme ne doit-elle pas rester au coeur d'une politique touristique digne de ce nom?

\section{La régionalisation du tourisme}

Lors de la Conférence socio-économique sur le tourisme en 1978, les associations touristiques régionales furent reconnues officiellement et consacrées comme les *interlocuteurs du ministère en régionsw. La dynamique régionale a nettement marqué le développement touristique au Québec: le lobby des régions est puissant, influençant lesorientations et les stratégies gouvernementales. Le tourisme a des retombées économiques et sociales évidentes pour les régions, mais toutes les régions ont-elles un potentiel touristique?

Ne faut-il pas plutôt miser sur les attractions, sur les produits les plus susceptibles d'attirer les touristes étrangers quitte à avantager nettement certaines régions au plan des budgets de promotion et de développement de l'offre? Cette remise en question ne fait cependant pas l'unanimité surtout que l'Etat québécois semble vouloir insuffler un dynamisme nouveau aux régions avec les conseils régionaux de développement (CRD) et l'action du Secrétariat aux affaires régionales (SAR). Pour plusieurs régions, le tourisme demeure ou devient l'une des pierres angulaires de la politique économique régionale.

\section{La protection du consommateur}

Les années 1970 furent celles des lois de protection du consommateur. Le secteur du tourisme n'y a pas échappé vu la 


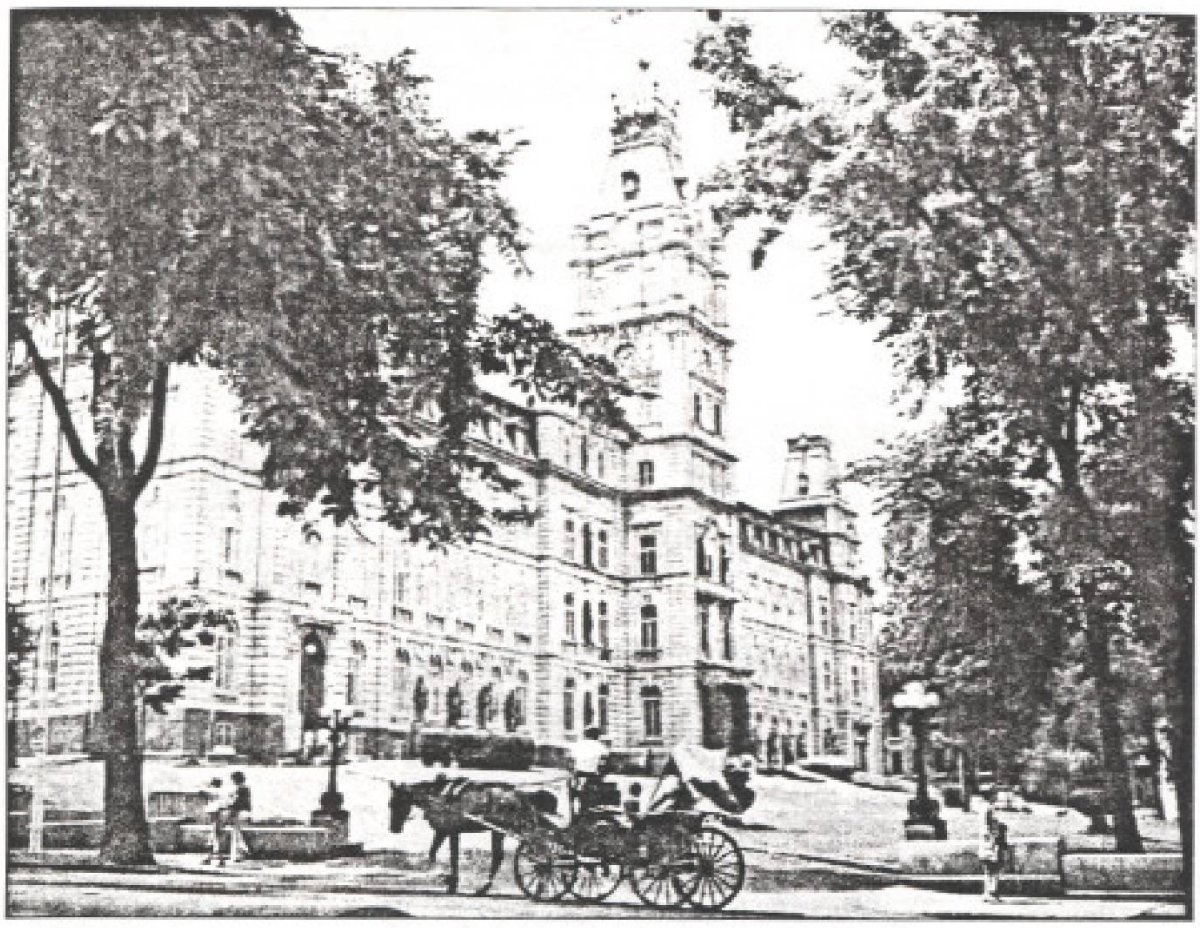

vulnérabilité particulière du consommateur touristique face aux fournisseurs de services. La Lơ sur Ibôtellerie ou la Loi sur les établissements touristiques qui l'a remplaoée, en 1991, de même que la Loisur les agents de voyages exigent la détention d'un permis pouropérer, obtenu à certaines conditions, et imposent à son détenteur un certain nombre d'obligations. Globalement, la tendance demeure favorable au consommateur même si les coupures dans les budgets de l'État ont réduit le nombre d'inspecteurs chargés de l'application des lois. Le relais fut pris par les tribunaux qui n'hésitent plus à rendre responsables les fournisseurs de services, les grossistes et mëme les agences de voyages détaillantes. La professionnalisation du tourisme

Secteur créateur d'emplois, mais reconnu aussi pour ses emplois peu qualifiés. L'État québécoois a investi dans la formation des ressources humaines par la création, il $y$ a vingt-cing ans, de l'Institut de tourisme et d'hồtellerie du Québec, par la création de programmes dans plusieurs Cégeps, par l'ouverture à l'UQAM en 1978 du baccalauréat en gestion et intervention touristiques... sans compter les programmes de niveau secondaire et ceux de l'éducation permanente et de la formation sur mesure. Maisn'ya-t-il pas tropde programmes? $\mathrm{La}$ formation sur mesureest ầl'honneur (grầce aux cuédits d'impôt!') et devient lucrative pour de nombreuses entreprises publiques et privées. Cette prolifération des programmes n'a pas nécessairement entraîné une réelle reconnaissance des diplômés sur le marché de l'emploi. La professionnalisationdu tourisme passenon seulement par la formation mais aussi par l'embauche d'un personnel bien formé et qualifié.

\section{La consultation et la concertation des partenaires}

Dès 1932, lorsqu'on donna une structure au service du tourisme établi sept ans auparavant, on créa aussi un Conseil du tourisme. Ce fut une première manifestation de la volonté del'Etat de consulter les acteurs du secteur privé. Prétextant l'impérạtif de réduire le nombre d'organismes d'Etat, le Conseil québécois du tourisme fut aboli par le gouvernement libéral au milieu des années 1980. Sur diverses questions, l'État consulte directement les associations sectorielles. Ces dernières années, le ministère du Tourisme a fait participer des représentants du secteur privé sur des comités de produits qu'il a mis en place en conformité avec les orientations du Plan marketing du ministère et de la Politique du tourisme. Une impression demeure cependant: l'absence de concertation générale au niveau de l'ensemble de l'industrie. Defaçon conjoncturelle, sur des questions d'actualité (taxation, casinos...), une coalition de l'industrie touristique du Québec s'est formée pour défendre des positions communes. Mais il n'y a rien de permanent!

\section{Le tourisme comme secteur d'intervention de l'État}

L'État a plusieurs raisons d'intervenir dans le secteur du tourisme, Malgré ces raișons, des décisions de l'État québécois (ex.: certaines privatisations réalisées ou en cours), révèlent que le tourisme est un secteur économique qui doit évoluer selon les règles de jeu du marché. Même sii un mora toire a été décrété au début 1994 sur les projets de privatisation des parcs québécois, plusieurs contrats de gestion ont été conclus ces dernières années avec l'entreprise privée. On assiste donc à un retrait de l'Etat, du moins de la gestion des biens collectifs. Les budgets de fonctionnement des ministères sont à la baisse, reflétant ainsi la volontế de l'État de ne pas augmenter son déficit à défaut de le réduire. $\mathrm{Par}$ contre, on continue à soutenir financièrement le développement de l'offre et de la promotion touristiques!

Le tourisme est un secteur de la vie économique qui doit évoluer selon les règles du marché.... avec un coup de pouce de l'État?? Le tourisme est un secteur de la vie sociale qui doit se plier aux exigences économiques!?... A trout considérer, on assiste au retrait de l'Etat mais celui-ci se retire du bout des pieds avecl'envie d'y revenir. Les caractéristiques particulières du tourisme sont telles qu'il ne peut en être autrement: les raisons de son intervention, de caractère social, économique, culturel, sont toujours là et devrąient continuer de justifier la présence de l'État. $\boldsymbol{f}$

\section{NOTES}

(1) Les auteurs veulent soulgner que le trawail de documentation a la base de cel artide a de réalise dans le cadre des travaux du 1" Colloque syndical sur l'industrie touristique, organisé par la Féderation du Commerce CSN, et tenu a Montreal en novembre 1994. Les auteurs faisaient partie de requipe de recherche de IUUCAM, assocte au projet dans le cadre du protocole d'entente UOAM CSN-FTO

(2) Ce tableaus inspire d' un texte de Jacques Demers, L'evolution du tourisme quebecois a travers les politiques qui front faponne, dans Lea politiques touristiques. Actes du colloque Teoros, Mon. treal, Teoros foollection Colloques et congres no 11. 1991 , pp. 13 a 16 .

(3) Nous omettons volontairement de parter de l"twolution de certaines structures politiques ou publi. ques qui ont aussi une incidence sur le phenomene touristique, mais de façon moins structu. rante que les ministëres et organismes d'État retenus dans ce tableau.

(4) L'article de monsieuf Jacques. Demers datant de 1991 , le lecteur voudra bien noter que nous prenons la liberté de nommer ainsi la sixieme periode du tourisme qui devrait d'ailleurs se poursuivre jusqu'd lorée de lan 2000. 